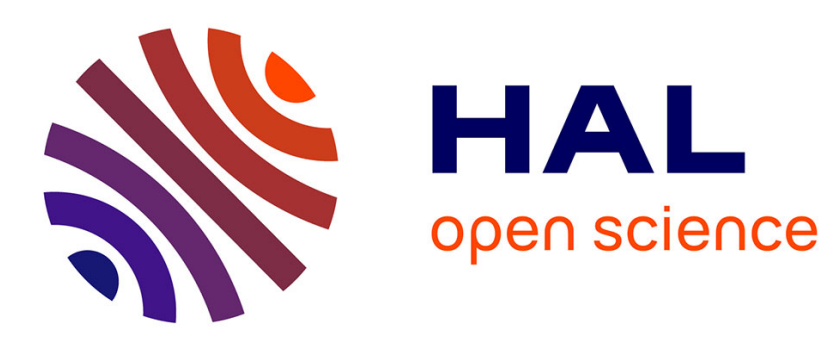

\title{
Etude de l'interface (211) $\Sigma 3$ dans le silicium par la méthode des franges alpha
}

\author{
A. Rocher, M. Labidi
}

\section{To cite this version:}

A. Rocher, M. Labidi. Etude de l'interface (211) $\Sigma 3$ dans le silicium par la méthode des franges alpha. Revue de Physique Appliquée, 1986, 21 (3), pp.201-205. 10.1051/rphysap:01986002103020100 . jpa00245431

\section{HAL Id: jpa-00245431 https://hal.science/jpa-00245431}

Submitted on 1 Jan 1986

HAL is a multi-disciplinary open access archive for the deposit and dissemination of scientific research documents, whether they are published or not. The documents may come from teaching and research institutions in France or abroad, or from public or private research centers.
L'archive ouverte pluridisciplinaire HAL, est destinée au dépôt et à la diffusion de documents scientifiques de niveau recherche, publiés ou non, émanant des établissements d'enseignement et de recherche français ou étrangers, des laboratoires publics ou privés. 
Classification

Physics Abstracts

$61.16 \mathrm{D}$

\title{
Etude de l'interface (211) $\Sigma \mathbf{3}$ dans le silicium par la méthode des franges alpha
}

\author{
A. Rocher et M. Labidi (*) \\ Laboratoire d'Optique Electronique, 29, rue Jeanne Marvig, F-31400 Toulouse, France \\ * Département de Physique, Faculté des Sciences, Tunis, Tunisie
}

(Reçu le 21 novembre 1984, révisé le 23 octobre 1985, accepté le 29 novembre 1985)

\begin{abstract}
Résumé. - L'étude du joint (211) $\Sigma 3$ est faite dans le silicium en microscopie électronique en transmission à l'aide de la technique des franges alpha. La précision de la détermination du vecteur translation rigide $\mathbf{T}$ a été améliorée en utilisant des conditions d'observation particulières. Le vecteur $T$ ainsi déterminé est caractérisé par $(0,08 \pm 0,01)$ $[1 \overline{1} 1]+(0,06 \pm 0,01)[21 \overline{1}]$. La composante suivante [011] a été trouvée égale à 0.
\end{abstract}

\begin{abstract}
Sigma 3$ has been studied in silicon by means of the alpha fringes technique in transmission electron microscopy. The accuracy in determining the rigid body translation $\mathbf{T}$ has been improved by using special observation conditions. This vector has been characterized as $(0.08 \pm 0.01)[1 \overline{1} 1]+(0.06 \pm 0.01)$ [21 1$]$. The components along [011] have been found equal to 0 .
\end{abstract}

\section{Introduction.}

Le but de cet article est de montrer que la méthode des franges $\alpha$, proposée par Pond et Smith [1], voit ses performances nettement améliorées par l'utilisation de conditions d'observations particulières. Dans le cas des joints de grains de coïncidence, cette méthode a pour objet de déterminer la position relative des deux grains au niveau de l'interface. Cette position relative, définie par un vecteur déplacement $\mathbf{T}$, constitue la relaxation du premier ordre des deux grains par rapport à leur position de coïncidence. Sa détermination se fait à l'aide de systèmes de franges équivalents à ceux associés aux défauts d'empilement (franges $\alpha$ ).

Cette étude est faite sur le joint de grains (211) $\Sigma 3$ dans le silicium. Ce joint est caractérisé par le plan d'interface $(21 \overline{1})$ associé à la désorientation (011, $\left.70.53^{\prime \prime}\right)$ qui correspond en notation de coïncidence à un indice $\Sigma 3$. L'intérêt de ce joint est de constituer un cas d'école. En effet, la détermination de $\mathbf{T}$ par la méthode des franges $\alpha$ est réellement envisageable car il est possible d'observer plus de trois systèmes de franges associés à des vecteurs de diffraction communs aux deux grains. Cette translation a déjà été étudiée dans le silicium par Fontaine et Smith [2], Pond et Vlachavas [3] ainsi que par Labidi [4] et dans le germanium par Bacmann [5] et par Bourret et al. [6].
Les résultats présentés ici précisent et complètent ceux de Fontaine et Smith [2] et confirment ceux de Bourret et al. [6].

\section{Principe de la détermination de $T$.}

Lorsque l'image est obtenue en condition de deux ondes avec une réflexion $\mathbf{G}_{\mathrm{c}}$ commune aux deux grains, le joint peut fournir un système de franges strictement équivalent à ceux observés pour un défaut d'empilement. Un tel contraste met en évidence une discontinuité des plans réflecteurs $\mathbf{G}_{\mathrm{c}}$ au niveau de l'interface. L'ensemble de ces discontinuités est caractérisé par un vecteur déplacement $\mathbf{T}$ connu sous la dénomination anglaise de rigid body translation. Le produit $\mathbf{G}_{\mathrm{c}} * \mathbf{T}$ correspond au déphasage introduit par le défaut entre le faisceau diffracté et le faisceau transmis dans les relations de la théorie dynamique. Il est directement relié à la valeur des contrastes associés au joint. La détermination de $\mathbf{T}$ se fait à l'aide de l'évaluation des produits $\mathbf{G}_{\mathrm{c}} * \mathbf{T}$ à partir de trois systèmes de franges obtenus avec des vecteurs de diffraction non coplanaires.

Dans le cas de la relation de coïncidence $\Sigma 3$, il existe huit vecteurs de diffraction $\langle 111\rangle,\langle 220\rangle$ et $\langle 311\rangle$ communs aux deux grains, représentés sur la projection stéréographique de la figure 1 . 


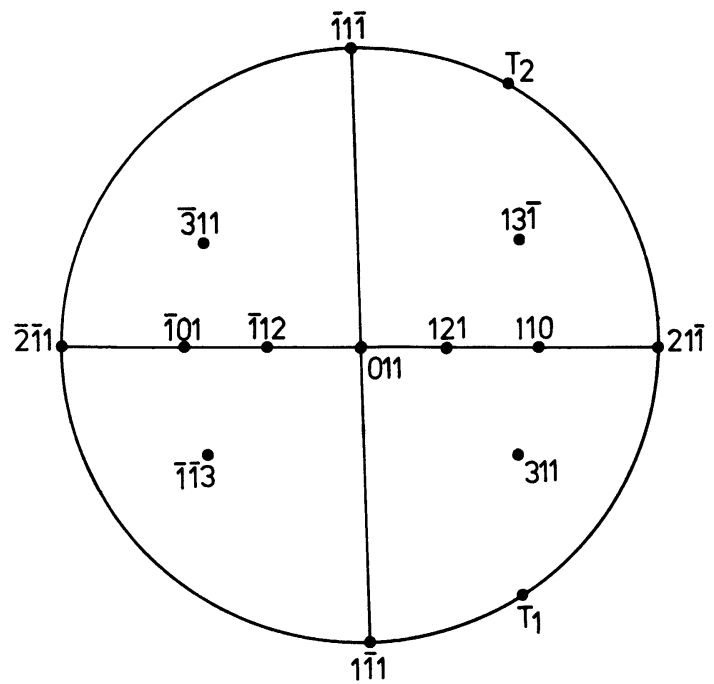

Fig. 1. - Projection stéréographique sur laquelle sont représentées : les directions du cristal de référence communes aux deux grains et se correspondant dans la relation de macle $\Sigma 3$ : les vecteurs déplacement $T_{1}$ et $T_{2}$ qui caractérisent la position relative des deux grains de part et d'autre des deux interfaces D1 et D2.

[Stereographic projection : common directions related to the $\Sigma 3$ twin relationship are set in the crystal taken as referential. Rigid body translations $T_{1}$ and $T_{2}$, characterizing the relative position of the grains in the interfaces D1 and D2.]

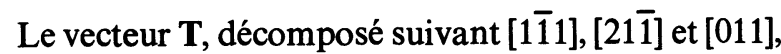
s'écrit :

$$
\mathbf{T}=x\left[\begin{array}{ll}
1-1 & 1
\end{array}\right]+y\left[\begin{array}{lll}
2 & 1-1
\end{array}\right]+z\left[\begin{array}{lll}
0 & 1 & 1
\end{array}\right] .
$$

Le produit scalaire $\mathbf{G}_{\mathrm{c}} * \mathbf{T}$, d'un vecteur diffraction commun aux deux grains $\mathbf{G}_{\mathrm{c}}$ par le vecteur déplacement $\mathbf{T}$ s'écrit :

$$
\mathbf{G}_{\mathrm{c}} * \mathbf{T}=2 \pi * g t .
$$

Dans cette équation le paramètre de déphasage $g t$ a une valeur comprise entre 0 et 1 . Les expressions littérales de $g t$ sont données en fonction de $x, y$ et $z$ sur le tableau I pour les vecteurs de diffraction utilisés dans cette étude.

\begin{tabular}{|c|c|c|c|}
\hline & $\mathbf{G}_{\mathrm{c}}$ & $\mathbf{G}_{\mathrm{c}} * \mathbf{T}=2 \pi * g t$ & $g t$ estimé \\
\hline \multicolumn{4}{|c|}{ Axe de zone [1112] I-II } \\
\hline $\begin{array}{l}1 \\
3\end{array}$ & $\begin{array}{ll}-1 & 1 \\
-3 & 3\end{array}$ & $\begin{array}{l}3 * x \\
9 * x\end{array}$ & $\begin{array}{r}0,3 \pm 0,1 \\
-0,3 \pm 0,1\end{array}$ \\
\hline 2 & 20 & $6 * y$ & $0,3 \pm 0,1$ \\
\hline $\begin{array}{r}1 \\
-3\end{array}$ & $\begin{array}{rr}3 & -1 \\
-1 & -1\end{array}$ & $\begin{array}{l}-3 * x+6 * y+2 * z \\
-3 * x-6 * y-2 * z\end{array}$ & $\begin{array}{l}0,1 \pm 0,05 \\
0,3 \pm 0,1\end{array}$ \\
\hline \multicolumn{4}{|c|}{ Axe de zone [121] II-I } \\
\hline 2 & $0-2$ & $6 * y-2 * z$ & $-0,3 \pm 0,1$ \\
\hline $\begin{array}{r}-1 \\
3\end{array}$ & $\begin{array}{rr}-1 & 3 \\
-1 & -1\end{array}$ & $\begin{array}{l}3 * x-6 * y+2 * z \\
3 * x+6 * y-2 * z\end{array}$ & $\begin{array}{l}-0,1 \pm 0,05 \\
-0,3 \pm 0,1\end{array}$ \\
\hline
\end{tabular}

Tableau I.
Le principal problème est d'estimer à partir des systèmes de franges ces produits $\mathbf{G}_{\mathrm{c}} * \mathbf{T}$. La précision de la détermination de ces produits par l'interprétation directe des observations est limitée par les incertitudes concernant les paramètres d'absorption et les phénomènes de fond continu [7]. Elle ne peut être meilleure que $30 \%$ pour les systèmes de franges à fort contraste. Elle sera moins bonne pour les faibles contrastes.

Aussi les valeurs de $g t$, reportées sur le tableau I, ont été estimées expérimentalement en associant aux systèmes de franges présentant :

- des contrastes de franges $\pi$, une valeur comprise entre 0,4 et 0,6 ;

- des contrastes importants, équivalents à ceux observés pour les défauts d'empilement, une valeur comprise entre 0,2 et 0,4 ;

- des contrastes faibles mais visibles comme dans le cas des réflexions $\{311\}$, une valeur comprise entre 0,05 et 0,15 ;

- des contrastes nuls, une valeur inférieure à 0,05 ;

- le signe du produit $g t$ est déterminé par la nature de la frange extérieure.

\section{Résultats expérimentaux.}

Les échantillons observés ont été prélevés dans des lingots polycristallins de silicium "SILSO » de Wacker. Polis mécaniquement jusqu'à une épaisseur de l'ordre de 100 microns. Ils sont ensuite amincis par polissage mécanochimique avec une solution de nitrate de cuivre et de fluorure d'ammonium pour rendre leur épaisseur inférieure au micron [8]. Les observations ont été faites sur un JEOL $200 \mathrm{CX}$ à $200 \mathrm{kV}$.

La planche 1 présente les micrographies utilisées pour cette étude. L'épaisseur de l'échantillon a été déterminée à la fois géométriquement et par le nombre de franges au niveau de l'interface. Elle est de l'ordre de $0,5 \mu \mathrm{m}$.

Une attention particulière a été apportée pour que les systèmes de franges soient vraiment spécifiques des défauts d'empilement. Pour celà, il a fallu vérifier que les conditions de deux ondes en position de Bragg étaient bien les mêmes pour les deux grains. La symétrie des systèmes de franges en fond clair et l'égalité des intensités des fonds continus ont été utilisées comme critères de bonne condition d'observation. Lorsque ces critères sont vérifiés les deux grains ont les mêmes conditions de diffraction et se comportent donc comme un cristal unique contenant un défaut d'empilement.

Il faut noter ici un point particulier relatif à la géométrie de l'échantillon. La surface d'entrée de l'échantillon a sa normale voisine de [011] contenue dans le joint. Lorsque la position relative du faisceau d'électrons par rapport à l'échantillon passe d'un azimuth proche de [112] à un autre voisin de [121], les électrons voient les deux grains dans un ordre différent. Il y a donc, entre les deux conditions d'observation, un changement de repère de référence où est 

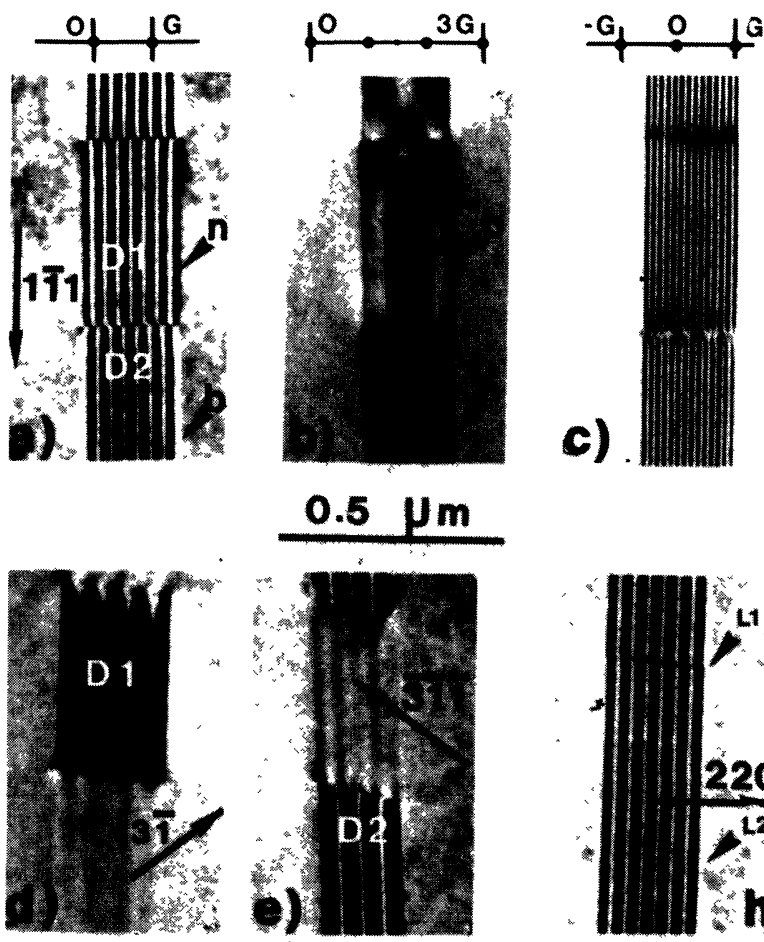

\section{$0.5 \mathrm{Jm}$}
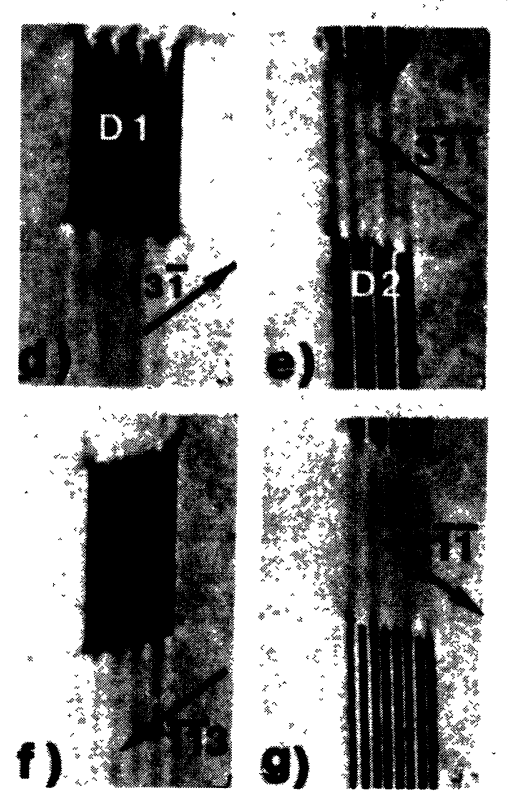
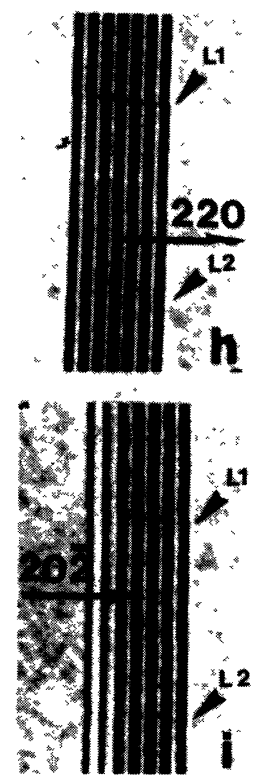

Planche 1. - Observations à $200 \mathrm{kV}$ des contrastes associés à l'interface (211) $\Sigma 3$. a) $s g=0$, contraste classique du domaine D1 avec les franges extérieures noires (notée $\mathrm{n}$ ). b) $s 3 g=0$, les franges extérieures associées à cette condition de diffraction sont blanches (notée b). c) pour la condition symétrique de diffraction les systèmes de franges sont les mêmes pour les deux domaines. d) et f) contraste important du domaine D1. e), g) faible contraste du domaine D1. h) i) les systèmes de franges sont les mêmes pour les deux domaines. Les contrastes associés aux limites de domaines L1 et L2 sont différents.

[TEM observations at $200 \mathrm{keV}$ of (211) $\Sigma 3$ interface. a) $s g=0$, classical contrast of D1 with black external fringes (noted n). b) $s 3 g=0$, external fringes are white (noted b). c) The systems of fringes observed for symmetrical diffraction conditions are equivalent in both domains. d, f) Strong contrast of D1. e, g) Weak contrast of D1. $h$, i) Both domains yield the same system of fringes. The contrasts observed at the limit of the domains L1 and L2 are different. 7

mesuré T. Dans le premier cas $\mathbf{T}$ mesure le déplacement du grain II par rapport au grain $I$ alors que dans le second cas $\mathbf{T}^{\prime}$ donne le déplacement du grain I par rapport au grain II. Il y a donc changement du

signe de $\mathbf{T}$ et donc de la nature des systèmes de franges. Pour conserver le même référentiel pour les deux positions de faisceau il suffit donc de changer le signe de $\mathbf{T}$ dans la seconde position.

Les micrographies réalisées avec les plans réflecteurs [1111] et $\{311\}$ font apparaître sur le même joint deux systèmes de franges distincts (planche $1 \mathrm{a}, \mathrm{d}$. e, $\mathrm{f}$, g). Ces systèmes mettent en évidence que les produits $\mathbf{G}_{\mathrm{c}} * \mathbf{T}$ ne sont pas équivalents et donc qu'il existe au moins deux domaines. D1 et D2, caractérisés par des vecteurs de déplacement T1 et T2 différents.

La réflexion (11̄1) fait apparaître sur le joint, planche 1a, deux systèmes de franges de natures différentes qui montrent que les produits $g t$ ne sont pas de même signe. Dans ces conditions, les composantes de $\mathbf{T 1}$ et $\mathbf{T} 2$ dans la direction (111) sont de signes opposés. La condition d'orientation symétrique du faisceau d'électrons par rapport au cristal utilisée pour la réflexion (111) fournit un même système de franges pour les deux domaines (planche 1c). Les deux composantes suivant [1111] sont donc égales en module mais de signes opposés.

Les réflexions (220) et (202) fournissent un même système de franges pour les deux domaines (planche $1 \mathrm{~h}, \mathrm{i})$. Les composantes suivant [011] et [211] des deux vecteurs $\mathbf{T 1}$ et $\mathbf{T} 2$ sont donc strictement égales.

Ces observations permettent de conclure que les composantes des vecteurs $\mathbf{T} 1$ et $\mathbf{T} 2$ sont égales en module et qu'elles sont de même signe suivant [011]

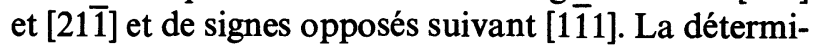
nation de $\mathbf{T}$ se fera en discutant le comportement des systèmes de franges observés pour un seul des domaines, D1 par exemple.

\section{Détermination des composantes de $\mathbf{T}$.}

Cette détermination revient à évaluer les trois paramètres $x, y$ et $z$ définis dans la relation (1). La valeur de $x$ est d'abord étudiée à l'aide du contraste associé à la réflexion (11̄1). Les réflexions $\{311\}$ fournissent les indications nécessaires à l'évaluation de $y$ et $z$.

La réflexion (111), dont le produit $g t$ est égal à $3 * x$, fournit un système de franges spécifique de la composante [1iㅣ]. Le contraste important, observé sur la micrographie a) de la planche 1 , permet d'attribuer à $g t$ une valeur comprise entre $-0,2$ et $-0,4$, c'est-à-dire :

$$
x=-0,11 \pm 0,03 .
$$

C'est le résultat donné par Fontaine et Smith, complété par l'incertitude de la mesure. Pour améliorer cette détermination, nous avons comparé les systèmes

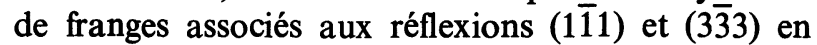
position de Bragg exacte. Il est bien établi que les franges extérieures, observées en champ clair, sont blanches quand $g t$ est positif et noires dans le cas contraire. En appliquant cette règle aux deux systèmes de franges $g$ et $3 g$ il est possible de déterminer si $x$ est supérieur, égal, ou inférieur à $1 / 9$. Les paramètres 
de déphasage associés à ces deux réflexions sont donnés par :

$$
g t_{1}=3 * x \quad \text { et } g t_{3}=9 * x
$$

selon la valeur de $x$, trois cas peuvent se présenter

1) $x=1 / 9: g t_{1}=0,33$ et $g t_{3}=1$.

Cette valeur donnée à $g t_{3}$ correspond à un déphasage nul. Le système de frange $3 g$ ne doit faire apparaître aucun battement associé à l'interférence $0,3 \mathrm{~g}$.

2) $x>1 / 9: 0,33<g t_{1}<0,4$ et $0<g t_{3}<0,2$.

Ces deux valeurs de $g t$ conduisent toutes deux à un déphasage de même signe. Les systèmes de franges $g$ et $3 g$ doivent avoir la même nature de frange extérieure.

3) $x<1 / 9: 0,2<g t_{1}<0,33$ et $-0,4<g t_{3}<0$.

Les déphasages sont dans ce cas de signes opposés. Les systèmes de franges $g$ et $3 g$ doivent être de natures différentes. Les franges extérieures sont alors claires pour l'un des systèmes et sombres pour l'autre.

Les micrographies a) et b) de la planche 1 imagent les systèmes de franges associés aux deux conditions de diffraction (111) et $(3 \overline{3} 3)$ en position de Bragg. Dans le premier cas, le domaine D1 présente des franges extérieures qui sont noires. La micrographie b) fait apparaître deux systèmes de franges : le premier, à faible période, caractérise l'interaction du premier ordre; le second caractérise l'interaction $(0,3 \overline{3} 3)$ avec une distance d'extinction de l'ordre de $200 \mathrm{~nm}$. Les franges extérieures associées à ce système sont claires.

D'après la discussion précédente, $x$ est donc strictement inférieur à $1 / 9$ puisque les franges extérieures associées aux deux systèmes étudiés sont différentes. De plus, le contraste observé pour le système de franges associé à la réflexion ( $3 \overline{3} 3$ ) est positif et assez important pour donner à $9 * x$ une valeur comprise entre 0,2 et 0,4 . Ces deux limites sont du fait de la définition du déphasage à $2 * \pi$ près, équivalentes à $-0,6$ et $-0,8$, ce qui donne :

$$
x=-0,08 \pm 0,01 \text {. }
$$

L'estimation est ici trois fois plus précise que par l'étude du contraste associé à la réflexion (11̄1), car si l'incertitude sur l'évaluation du déphasage demeure la même, le déphasage est, lui, multiplié par 3.

Les deux autres composantes sont estimées avec une bonne précision à l'aide des contrastes associés aux réflexions $(13 \overline{1})$ et $(\overline{113})$. Les deux systèmes de franges présentent un faible contraste avec des franges extérieures blanches qui permettent de limiter les deux paramètres de déphasage $g t$ à des valeurs comprises entre 0,05 et 0,15 . Les expressions littérales donnant le déphasage s'écrivent en tenant compte du changement de signe de $\mathbf{T}$ pour la réflexion (13) comme cela a été discuté précédemment :

$$
\begin{aligned}
& 3 * x-6 * y+2 * z=0,1 \pm 0,05 \\
& 3 * x-6 * y-2 * z=0,1 \pm 0,05
\end{aligned}
$$

Ce système résolu en fonction de $x$, fournit les relations suivantes :

$$
\begin{gathered}
y=-x / 2+0,1 / 6 \pm 0,05 / 6 \\
z=0 \pm 0,025
\end{gathered}
$$

En prenant $x$ égal à $-0,08 \pm 0,01$ on trouve :

$$
y=0,06 \pm 0,01 \text {. }
$$

La comparaison des faibles contrastes a ainsi permis de mettre en évidence, avec une assez grande précision, que la composante de $\mathbf{T}$ suivant [011] est nulle. Il a fallu, pour cela, interpréter correctement les contrastes en remarquant notamment que le passage du faisceau de l'axe [112] à l'axe [121] changeait l'ordre des grains vus par les électrons et donc inversait le sens des vecteurs translations. $\mathrm{Si}$ nous n'avions pas tenu compte de ce fait nous aurions trouvé une composante suivant [011] non négligeable.

Le calcul de $y$ et $z$ à partir des réflexions $\{220\}$ donne un résultat un peu différent avec une erreur plus grande liée à l'incertitude absolue sur l'évaluation de $g t$ qui est ici supérieure à celle des réflexions [311].

Des essais de comparaison entre les systèmes de franges associés aux réflexions (220) et (660) ont aussi été réalisés. Des contrastes faibles et uniformes ont été observés pour la réflexion (660). Il n'a pas été possible de dire directement si ce comportement était lié à une valeur de $3 * g t$ proche de 0 ou à un effet dynamique de distance d'extinction trop importante. Cependant, la valeur de $y$ trouvée confirme a posteriori l'absence de contraste observée pour la réflexion (660).

\section{Limites d'interface.}

Les limites L1 et L2, bordant les domaines D1 et D2 et D2 et D1, sont portées par la direction [011]. L'absence de contraste oscillant observée pour les réflexions $(220)$ et $(\overline{202})$ (planches $1 \mathrm{~h}$ et i) permet de conclure que si l'on assimilait ces limites d'interface à des dislocations, elles auraient leur vecteur de Burgers porté par la direction [11ㅣ]. Cependant les contrastes résiduels associés aux deux limites ne sont pas équivalents. On observe pour L1 une largeur de contraste beaucoup plus importante que pour L2. Ceci indique que ces limites ne sont pas exactement de même nature. En particulier, il est probable que les deux changements de plans d'interface se font par l'intermédiaire de marche de largeur différente.

\section{Discussion.}

Les valeurs de $x, y$ et $z$ de la relation (1) sont reportées sur le tableau II. Elles sont à comparer aux valeurs déjà publiées.

Le vecteur déplacement $\mathbf{T}$, que nous avons déterminé grâce à l'utilisation de conditions d'observation particulières, sont un peu différents des résultats de Fontaine et Smith obtenus par cette même méthode à l'aide de critères de contraste simplifiés ( $g t$ égal à 0 
Tableau II.

\begin{tabular}{lccc} 
& $x[1 \overline{1} 1]$ & $y[21 \overline{1}]$ & $z[011]$ \\
\hline F et $\mathrm{E}[2]$ & 0,111 & 0,056 & 0 \\
\hline $\mathrm{V}$ et $\mathrm{P}[3]$ & 0,125 & 0,01 & 0 \\
\hline $\mathrm{L}[4]$ & 0,125 & 0,056 & 0 \\
\hline B, B et $\mathrm{P}[6]$ & $0,09 \pm 0,01$ & $0,05 \pm 0,02$ & 0 \\
\hline ce travail & $0,08 \pm 0,01$ & $0,06 \pm 0,01$ & $\pm 0,025$
\end{tabular}

ou 1/3) [2]. Ces valeurs sont cohérentes avec celles trouvées en microscopie à haute résolution par Bourret et al. dans le germanium [6] et donc compatibles avec le modèle totalement reconstruit que Papon et Petit [9] ont développé à partir d'observations en diffraction électronique.

La comparaison de deux systèmes de franges de faible contraste a aussi permis de montrer que les plans (011) étaient continus au niveau du joint.
Il faut noter enfin que, si l'on se réfère au modèle de Fontaine et Smith, la valeur de $x$ égale à $1 / 12$ fournit une structure d'interface symétrique. En effet, les deux cycles à 7 atomes prévus dans le modèle deviennent symétriques avec cette condition. Ce point peut être intéressant pour la minimisation de l'énergie de cet interface.

La méthode des franges $\alpha$ appliquée à l'étude des joints fournit des informations assez précises sur la position relative des grains au niveau de l'interface. L'utilisation de conditions d'observations particulières, faisant intervenir soit des réflexions d'ordre supérieur en position de Bragg soit des réflexions donnant de faibles contrastes, a permis de limiter l'incertitude des mesures à des valeurs inférieures à $0,02 \mathrm{~nm}$.

\section{Remerciements.}

Les auteurs remercient A. Bourret et J. J. Bacmann pour les discussions qu'ils ont eues à propos de cette étude, ainsi que L. Bernard pour son assistance technique.

\section{Bibliographie}

[1] Pond, R. C. et Smith, D. A., Canad. Met. Quart. 13 (1974) 1.

[2] Fontaine, C. et Smith, D. A., Appl. Phys. Lett. 40 (2) (1982) 153.

[3] Pond, R. C., J. Physique Colloq. 43 (1982) C1-51.

Vlachavas, D. S. et Pond, R. C., Inst. Phys. Conf. 60 (1981) 159.

[4] LabIDI, M., Thèse de Troisième Cycle, Faculté des Sciences, Tunis (1983).
[5] Bacmann, J. J., J. Physique Colloq. 43 (1982) C6-93.

[6] Bourret, A., Billard, L. et Petit, M., à paraître dans Microsc. Semicond. mat. Oxford, 1985.

[7] Rocher, A., Thèse d'Etat, Orsay (1973).

[8] Regh, J. et Silvay, G. A., J. Electrochem. Soc. 6 3-4 (1968) 155.

[9] Papon, A. M. et PetıT, M., Scripta Met. (in press) (1985). 